\title{
Divergent Signal-to-Noise Ratio and Stochastic Resonance in Monostable Systems
}

\author{
J. M. G. Vilar and J. M. Rubí \\ Departament de Física Fonamental, Facultat de Física, Universitat de Barcelona, Diagonal 647, E-08028 Barcelona, Spain
} (Received 7 June 1996)

\begin{abstract}
We present a class of systems for which the signal-to-noise ratio always increases when increasing the noise and diverges at infinite noise level. This new phenomenon is a direct consequence of the existence of a scaling law for the signal-to-noise ratio and implies the appearance of stochastic resonance in some monostable systems. We outline applications of our results to a wide variety of systems pertaining to different scientific areas. Two particular examples are discussed in detail. [S0031-9007(96)01331-2]
\end{abstract}

PACS numbers: $05.40 .+\mathrm{j}$

Stochastic resonance $(\mathrm{SR})[1-11]$ is a phenomenon wherein the response of a system to a driven periodic signal is enhanced at an optimized nonzero noise level. Although increasing the noise level in order to enable us to more easily detect a signal was considered counterintuitive, this constitutes one of the most surprising results of the SR. It seems obvious, however, that the signal-tonoise ratio (SNR) must go to zero as noise is increased indefinitely. Contrarily, in this Letter we present a class of systems in which the SNR always increases when the noise is increased and diverges at infinite noise level, instead of exhibiting a maximum at a particular value of the noise. This result implies the presence of SR in monostable systems for which a maximum in the SNR at nonzero noise level has never been observed before. These findings open up new possibilities concerning the application of SR to a great variety of physical, chemical, and biological systems. To be explicit, we have applied our results to two particular cases; namely, a ferromagnetic particle and a standard model of neural excitable medium.

The class of systems we will discuss is described by only one relevant degree of freedom whose dynamics is governed by the following Langevin equation:

$$
\frac{d x}{d t}=-h(t) x^{1+2 n}+\xi(t)
$$

where $h(t)=k\left[1+\alpha \sin \left(\omega_{0} t\right)\right]$, with $k$ and $\alpha(<1)$ constants, $n$ is an integer number, and $\xi(t)$ is Gaussian white noise with zero mean and second moment $\langle\xi(t) \xi(t+\tau)\rangle=D \delta(\tau)$, defining the noise level $D$. The system can be characterized by the quantity $v$ which is a function of the variable $x(t)$. This quantity is sometimes referred to as the response to the oscillating force. The effect of this force may be analyzed by the power spectrum

$$
P(\omega)=\int_{0}^{2 \pi / \omega_{0}} d t \int_{-\infty}^{\infty}\langle v(t) v(t+\tau)\rangle e^{-i \omega \tau} d \tau .
$$

To this purpose we will assume that it consists of a delta function centered at the frequency $\omega_{0}$ plus a function $Q(\omega)$ which is smooth in the neighborhood of $\omega_{0}$ and is given by $P(\omega)=Q(\omega)+S\left(\omega_{0}\right) \delta\left(\omega-\omega_{0}\right)$.
Then, the SNR is defined by $\mathrm{SNR} \equiv S\left(\omega_{0}\right) / Q\left(\omega_{0}\right)$ and, consequently, has dimensions of inverse of time.

The existence of a characteristic time $\tau$ in our system will enable us to propose the form of the SNR through the simple scaling law

$$
\mathrm{SNR}=f\left(\alpha, \omega_{0} \tau\right) \tau^{-1},
$$

where $\tau^{-1}=D^{n /(1+n)} k^{1 /(1+n)}$ and $f\left(\alpha, \omega_{0} \tau\right)$ is a dimensionless function, provided that $v(x)$ does not introduce another characteristic time. We will suppose that for a given value of $\tau$ the limit of SNR when $\omega_{0}$ goes to zero exists. As such, the following expression for small driving frequencies holds

$$
\mathrm{SNR} \approx f(\alpha, 0) \tau^{-1} .
$$

Let us now discuss the main characteristics of our model upon varying the exponent $n$. If $n=0$, one finds the exact result SNR $=f\left(\alpha, \omega_{0} k^{-1}\right) k$, which does not depend on the noise level. Even more interesting is the behavior obtained for the case $n>0$. The scaling of the SNR indicates that it increases when increasing the noise level, achieving the behavior $\mathrm{SNR} \propto D$ as $n$ goes to infinity. A particular and common situation illustrating this case corresponds to the potential $V_{1}(x)=$ $\frac{1}{4} h(t) x^{4} \quad$ [Fig. 1(a)], obtained when $n=1$, for which SNR increases as $\sqrt{D}$. In Fig. 1(b) we have depicted the SNR corresponding to $V_{1}(x)$. Here the magnitude giving the response of the system has been taken $v(x)=$ $x^{2}$. Our result is obtained from numerical simulations by integrating the corresponding Langevin equation by means of a standard second-order Runge-Kutta method for stochastic differential equations [12,13]. In order to verify the scaling law proposed through Eq. (4) we have fit the values of the SNR by a power law in the range of $D$ from 1 to 1000 for the potential $V_{1}(x)$. SNR is given by $a D^{b}$ with $b=0.498 \pm 0.004$ and $a=0.76 \pm 0.02$, which is in good agreement with the theoretical value $b=\frac{1}{2}$. The power spectrum corresponding to $V_{1}(x)$ for two values of the noise levels is shown in Fig. 1(c). Both the signal and noise background increase when increasing $D$, but the signal increases faster.

In spite of its simplicity, our model encompasses a great variety of common situations. Around an equilibrium 

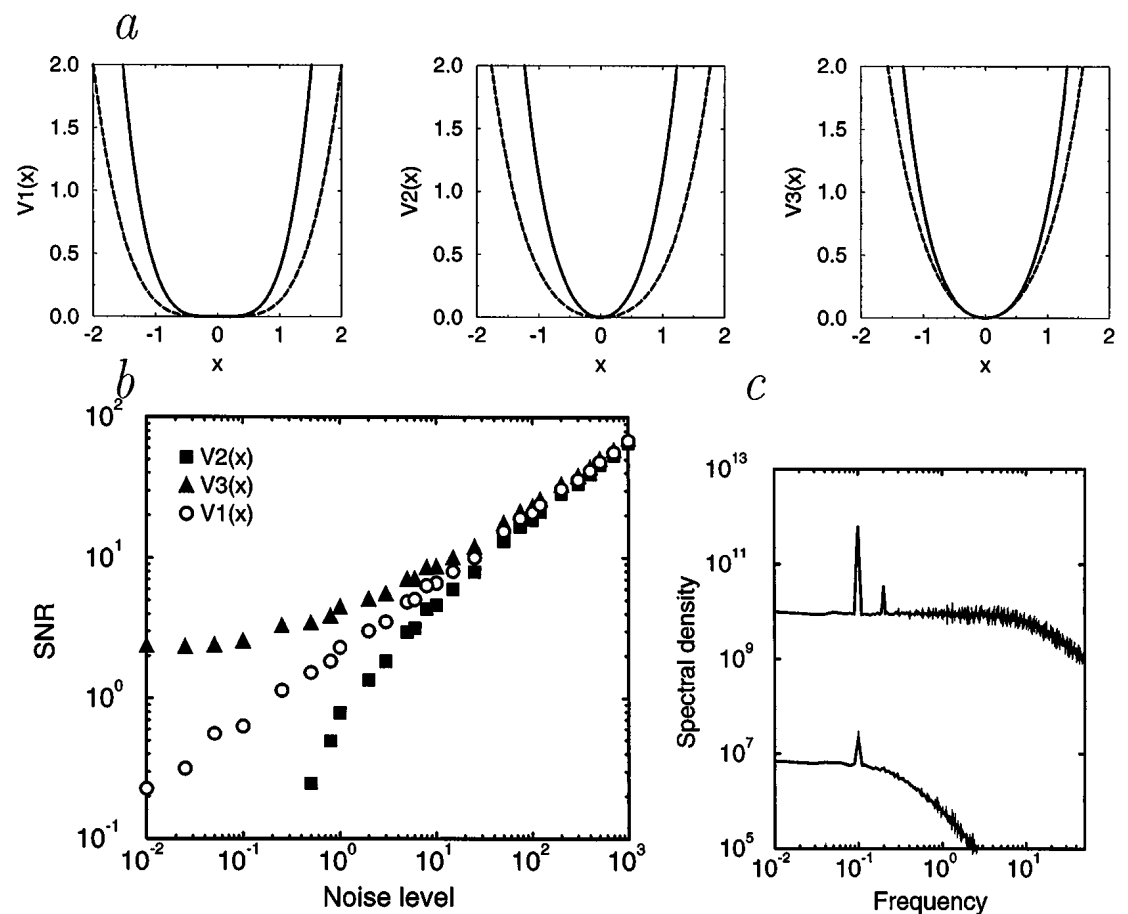

\section{$C$}

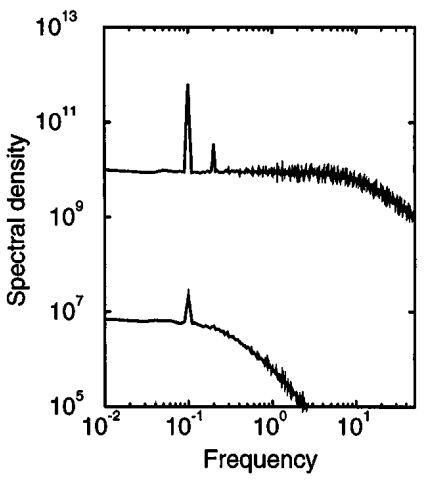

FIG. 1. (a) Potentials $V_{1}(x), V_{2}(x)$, and $V_{3}(x)$, for the maximum value of $h(t)$ (solid line) and for the minimum value (dashed line). Here $k=1, \alpha=0.5$, and $\omega_{0} / 2 \pi=0.1$. (b) Behavior of the SNR for the three potentials presented previously. (c) Power spectrum corresponding to $V_{1}(x)$ for $D=0.1$ and $D=1000$.

state most systems may be approximated by a parabolic potential. Thus for $n=0$ our model describes a system around an equilibrium state in a force field whose intensity varies periodically in time. A physical realization of such a system could be a dipole under an oscillating field. For $n=0$, however, the SNR is independent of $D$. In order to understand the behavior of the SNR, as $D$ is increased we must take into account the corrections to the parabolic approximation. Commonly, these corrections are given by a term proportional to $x^{4}$. Two particular realizations of this situation have been analyzed numerically resulting in an increase of the SNR [Fig. 1(b)] with the noise level. For $V_{2}(x)=h(t)\left(\frac{1}{2} x^{2}+\frac{1}{4} x^{4}\right)$ [Fig. 1(a)], which basically corresponds to a potential that around the minimum grows faster than a parabolic one, we expect that the SNR is an increasing function of the noise, since for low noise level the potential behaves as $\frac{1}{2} h(t) x^{2}$, whereas for high noise level as $\frac{1}{4} h(t) x^{4}$. A slightly different potential is $V_{3}(x)=\frac{1}{2} x^{2}+\frac{1}{4} h(t) x^{4}$ [Fig. 1(a)], which differs from the previous one only in the behavior at low noise level. Since under this circumstance this potential reduces to $\frac{1}{2} x^{2}$ and, consequently, it is not modulated by $h(t)$, SNR goes to zero for low $D$. For $n=1$, our model describes the dynamics of a system at the critical point of both the pitchfork and Hopf bifurcations [14], occurring in many systems including, to mention just a few [15,16], chemical reactions, models of populations, convection in liquids, lasers, and instabilities in semiconductors.
The divergence of the SNR is due to the fact that the potential is unbounded. It is obvious that for a bounded potential the noise can completely destroy the response of the system. An important consequence follows from the previous results: If the SNR grows for low noise level (when the potential around the minimum can be approximated by a potential like $V_{2}$ or $V_{3}$ ) and the potential is bounded (which implies that SNR goes to zero for large noise) then the SNR exhibits a maximum, thus indicating the appearance of SR. To illustrate this point we report results of numerical simulations for the dynamics of a ferromagnetic particle [17-19] under an external magnetic field and with energy of anisotropy $h(t) \sin ^{4} \theta$, with $\theta$ being the angle between the magnetic moment and the axis of easy magnetization. The external magnetic field is then applied in the direction of the easy axis of magnetization and its intensity is as high as the system becomes monostable. The dynamics of the magnetization may be described by the Langevin equation

$$
\frac{d \theta}{d t}=-\sin \theta-h(t) \sin ^{3} \theta \cos \theta+\xi(t),
$$

where the first term on the right hand side accounts for the interaction with an external magnetic field, the second for the anisotropic effects, and the third is a noise source due to a random field or to thermal fluctuations. The parameter $h(t)$ is assumed to be oscillatory, the reason being, for example, the presence of oscillations of the pressure of the medium surrounding the particle. The response of the system is now given by $\cos \theta$, i.e., by 
the magnetization. For low noise, the potential [Fig. 2(a)] and the magnetization reduces to $\frac{1}{2} \theta^{2}+\frac{1}{4} h(t) \theta^{4}$ and $1-\frac{1}{2} \theta^{2}$, respectively. Therefore our previous results apply to this case. The corresponding SNR is shown in Fig. 2(b) and exhibits a maximum at a finite noise level. This result clearly shows the existence of an optimal noise level for which the system is more sensible to periodic changes of the environment. In Fig. 2(c) we have represented the power spectrum for some values of the noise level. Measurements of the SNR of a ferromagnetic particle under an external magnetic field and with energy of anisotropy have been performed in Ref. [20]. Although the experimental conditions are slightly different from the ones of our example a maximum in the SNR is also found. Further experiments, similar to the ones reported in [20], could be carried out to corroborate the remaining predictions of our model.

The next example to be considered corresponds to a standard model of a neural excitable medium. This model characterizes the activity generated in a slab of neural tissue comprising a very large number of closely packed and coupled nerve cells [21-23]. We will consider the case of all-to-all connectivity in which spatial dependence may be ignored. The model of neural excitable medium is given by the following equation [21]:

$$
C \frac{d U}{d t}=-R^{-1} U+\phi(U)+P,
$$

describing the dynamics of the spatial average of the transmembrane potential $U$. Here $C$ is the membrane capacitance, $R$ is the membrane resistance, and $P$ is an external current applied to the net. The nonlinear term $\phi(U)$ is proportional to the gain of the neuron and accounts for its mean firing rate. Its form is usually taken to be a sigmoidal, e.g.,

$$
\phi(U)=\varepsilon\left(1+e^{-\nu(U-\theta)}\right)^{-1},
$$

where $\nu$ is a constant, fixing the sensitivity to excitation of the population, $\theta$ is the threshold mean voltage, and $\varepsilon$ is a parameter depending on the structure of the net and the characteristics of the neuron. We will consider that the external current applied to the net fluctuates and that it may be approximated by a Gaussian white noise $[\langle P(t)\rangle=0$ and $\langle P(t) P(t+\tau)\rangle=D \delta(\tau)]$. The potential function [Fig. 3(a)] corresponding to the variable $U$ is given by

$$
V(U)=\frac{1}{C}\left(\frac{1}{2} R^{-1} U^{2}-\frac{\varepsilon}{\nu} \ln \left(1+e^{\nu(U-\theta)}\right)\right) .
$$

The membrane resistance $R$ can be modified through small changes in the permeability of a suitable ion. Let us consider that this modification is periodic in time. There exists a range of parameters for which the neurons of the net are not excited, i.e., $U=0$, consequently this state is not affected by small variations of $R$. The situation changes drastically with the addition of noise. For small

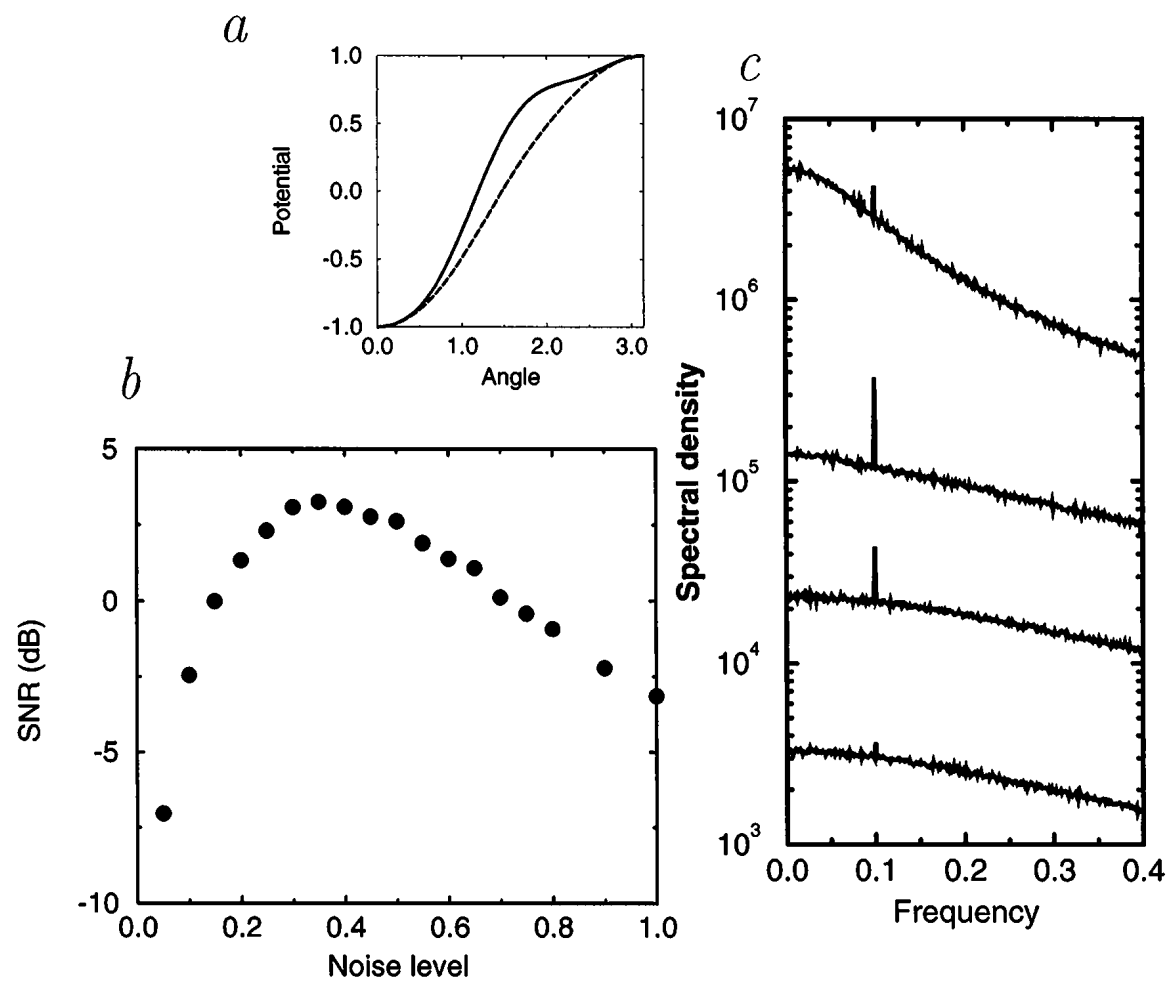

FIG. 2. (a) Potential energy of the ferromagnetic particle [Eq. (5)] as a function of $\theta$ for the maximum value of $h(t)$ (solid line) and for the minimum (dashed line). The parameters are taken $k=0.3, \alpha=3 / 2$, and $\omega_{0} / 2 \pi=0.1$. (b) SNR for the previous values of the parameters obtained through computer simulations. (c) Power spectrum for $D=0.05,0.15,0.35$, and 1 . 

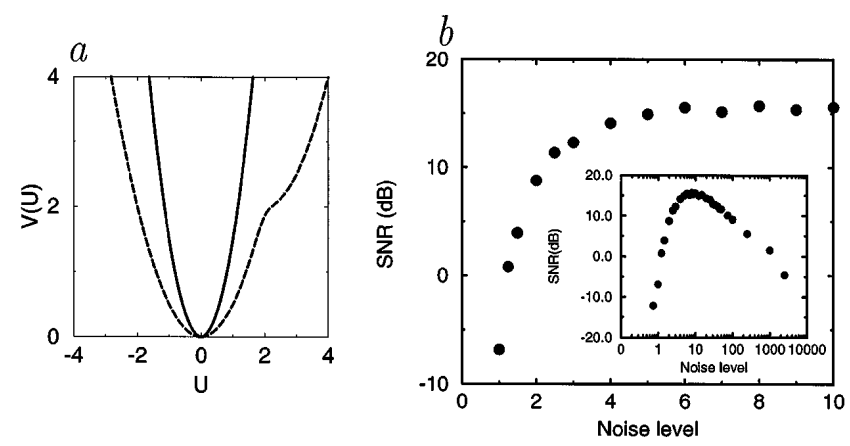

FIG. 3. (a) Representation of the potential function $V(U)$ for the maximum value of $R^{-1}=h(t)$ (solid line) and for the minimum value (dashed line). The parameters used here are $C=1, \theta=2, \varepsilon=1, \nu=10$, and the resistance $R^{-1}=h(t)$, with $k=2, \alpha=0.5$, and $\omega_{0} / 2 \pi=0.01$. (b) SNR for the previous parameters.

values of the noise, when the term $\phi(U)$ is irrelevant, the dynamics of this model may be described by Eq. (1), with $n=0$. In this case, the SNR for $v=U^{2}$ is independent of $D$, but in this system the interesting variable is $U$ instead of $U^{2}$. For small noise values, the potential is symmetric in $U$, thus giving a zero SNR for the variable $U$. When increasing the noise level the symmetry is lost, since positive fluctuations of $U$ may cause firing of the neurons. Variations of $R$ then modulate the amplitude of the fluctuations of $U$, giving rise to periodicity in the firing rate of the neurons, which implies a nonvanishing SNR. Further increasing of the noise level then leads to the restoration of the symmetry. Consequently, this model exhibits SR as has been shown in Fig. 3(b). It is interesting to remark that, in contrast with previous results concerning the appearance of SR for a single neuron $[24,25]$, our results refer to the prediction of the phenomenon for an ensemble of neurons.

Our analysis has revealed the presence of SR in a wide variety of situations, embracing different scientific areas, which have not been considered up to now. A methodological aspect to be emphasized, and that could be used in subsequent studies, is that arguments as simple as scaling laws or considerations about the symmetry may help us in predicting the enhancement of signals via SR. Our work, then, offers new perspectives on what concerns the consideration of SR as a general phenomenon that might apply to diverse systems.

The authors would like to thank A. Pérez-Madrid and Ricard V. Solé for fruitful discussions. This work was supported by DGICYT of the Spanish Government under Grant No. PB92-0859. One of us (J. M. G. V.) wishes to thank the Generalitat de Catalunya for financial support.
[1] R. Benzi, A. Sutera, and A. Vulpiani, J. Phys. A 14, L453-L457 (1981).

[2] S. Fauve and F. Heslot, Phys. Lett. A 97, 5-7 (1983).

[3] B. McNamara, K. Wiesenfeld, and R. Roy, Phys. Rev. Lett. 60, 2626-2629 (1988).

[4] B. McNamara and K. Wiesenfeld, Phys. Rev. A 39, 48544869 (1989).

[5] A. Simon and A. Libchaber, Phys. Rev. Lett. 68, 33753378 (1992).

[6] Proceedings of the NATO Advanced Research Workshop on Stochastic Resonance, San Diego, 1992 [J. Stat. Phys. 70, 1 (1993)].

[7] F. Moss, in Some Problems in Statistical Physics, edited by G. H. Weiss (SIAM, Philadelphia, 1994).

[8] K. Wiesenfeld, D. Pierson, E. Pantazelou, C. Dames, and F. Moss, Phys. Rev. Lett. 72, 2125-2129 (1994).

[9] K. Wiesenfeld and F. Moss, Nature (London) 373, 33 (1995).

[10] Z. Gingl, L. B. Kiss, and F. Moss, Europhys. Lett. 29, 191-196 (1995).

[11] F. Marchesoni, L. Gammaitoni, and A. R. Bulsara, Phys. Rev. Lett. 76, 2609-2612 (1996).

[12] P. E. Kloeden and R. A. Pearson, J. Aust. Math. Soc. B 20, 8-12 (1977).

[13] J. R. Klauder and W.P. Petersen, SIAM J. Numer. Anal. 22, 1153-1166 (1985).

[14] H. Schuster, Deterministic Chaos (VCH, Weinheim, 1989).

[15] H. Haken, Synergetics (Springer-Verlag, New York, 1983).

[16] J.D. Murray, Mathematical Biology (Springer-Verlag, New York, 1989).

[17] L.D. Landau and E. M. Lifshitz, Electrodynamics of Continuous Media (Pergamon, New York, 1981), Vol. 8.

[18] A. Peréz-Madrid and J. M. Rubí, Phys. Rev. E 51, 41594164 (1995).

[19] Y.L. Raikher and V.I. Stepanov, Phys. Rev. B 52, 3493 3498 (1995).

[20] A. N. Grigorenko, P.I. Nikitin, A. N. Slavin, and P. Y. Zhou, J. Appl. Phys. 76, 6335-6337 (1994); A.N. Grigorenko and P. I. Nikitin, Appl. Surf. Sci. 92, 466470 (1996).

[21] J.D. Cowan and G. B. Ementrout, in Studies in Mathematical Biology, edited by S. A. Levin (The Mathematical Association of America, Washington, 1978), Pt. I.

[22] H. R. Wilson and J. D. Cowan, Biophys. J. 12, 1-24 (1972).

[23] J. L. Fedelman and J. D. Cowan, Biol. Cybernet. 17, 3951 (1975).

[24] A. Longtin, A. Bulsara, and F. Moss, Phys. Rev. Lett. 67, 656-659 (1991).

[25] J. K. Douglass, L. Wilkens, E. Pantazelou, and F. Moss, Nature (London) 365, 337-340 (1993). 